\title{
Angèle Delaunois, Soledad du Soleil
}

\section{Simona Rossi}

\section{(Q) OpenEdition}

\section{Journals}

\section{Edizione digitale}

URL: https://journals.openedition.org/studifrancesi/39926

DOI: 10.4000/studifrancesi.39926

ISSN: 2427-5856

\section{Editore}

Rosenberg \& Sellier

\section{Edizione cartacea}

Data di pubblicazione: 1 décembre 2004

Paginazione: 423-424

ISSN: 0039-2944

\section{Notizia bibliografica digitale}

Simona Rossi, «Angèle Delaunois, Soledad du Soleil», Studi Francesi [Online], 143 (XLVIII | II) | 2004,

online dal 30 novembre 2015, consultato il 19 mai 2021. URL: http://journals.openedition.org/ studifrancesi/39926 ; DOI: https://doi.org/10.4000/studifrancesi.39926

Questo documento è stato generato automaticamente il 19 mai 2021.

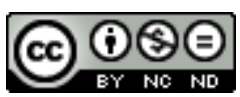

Studi Francesi è distribuita con Licenza Creative Commons Attribuzione - Non commerciale - Non opere derivate 4.0 Internazionale. 


\title{
Angèle Delaunois, Soledad du Soleil
}

\author{
Simona Rossi
}

\section{NOTIZIA}

ANGèle delaunoIs, Soledad du Soleil, Ottawa, Éditions Pierre Tisseyre, 2003, pp. 182.

1 Viviamo in un'epoca strana, inutile negarlo, fasulla ed esibizionista, che pare provare una soddisfazione quasi sadica nell'utilizzare l'infinita gamma delle emozioni umane per fare spettacolo. Ė così che travolti dal bombardamento quotidiano d'immagini più o meno strazianti, più o meno rispettose, noi uomini e donne del terzo millennio, abbiamo finito per perdere quanto possediamo di più prezioso, ossia le radici del nostro sentire più autentico e profondo, la spontaneità e la capacità di stupirci e di emozionarci. Tutto questo è rimasto intrappolato dietro ad una sorta d'involucro protettivo che si chiama indifferenza e che, consciamente o inconsciamente, quasi fosse un ulteriore, moderno dna, gli adulti finiscono per trasmettere ai giovani, ai bambini, agli adolescenti.

2 La casa editrice Pierre Tisseyre, punta di diamante nelle pubblicazioni quebecchesi di narrativa per ragazzi di tutte le età, da sempre specialista in questo settore, cerca d'infrangere proprio questo involucro, di andare oltre, di giungere al cuore di coloro che saranno gli adulti del domani. E lo fa attraverso romanzi di un'intelligenza e di un'intensità davvero eccezionali, romanzi per bambini e adolescenti, ma che forse possono insegnare qualcosa anche agli adulti.

3 Soledad du soleil è uno di questi. L'autrice propone la storia di un ragazzino canadese di appena sedici anni, Nicolas, che si reca con la madre a San Sebastián, nelle Antille francesi, per un breve periodo di vacanza. Qui, all'insaputa di Nicolas, s'incrociano due realtà adolescenziali completamente diverse: quella patinata, di serie $\mathrm{A}$, che hanno la fortuna di vivere i ragazzi dei paesi ricchi, figli del progresso e del benessere, e quella, ben più dolorosa, di serie $\mathrm{B}$, che subiscono invece i giovani dei paesi poveri, costretti a sopportare fin dalla più tenera età fame, soprusi e violenza. Incarnazione di questa infanzia violata e calpestata, di un'adolescenza praticamente inesistente, altri non è che 
Soledad, una piccola donna di soli tredici anni, una cerbiatta dai lunghi capelli corvini e dalla pelle color cannella che fa innamorare all'istante il giovane canadese. Tra quest'ultimo e questa sfortunata figlia delle Antille dagli occhi tristi, il cui nome significa peraltro solitudine, nasce quindi una tenera amicizia. Essa regalerà a Nicolas l'indescrivibile incanto del primo, acerbo innamoramento, ma senza rivelargli subito che una torbida verità, al di là di qualsiasi immaginazione, sporca la tela immacolata della sua ingenuità. Tutto il romanzo, così, è incentrato sul percorso di crescita del ragazzino, che scopre poco a poco, e con orrore crescente, la natura spesso ingannevole delle apparenze e la spaventosa realtà vissuta da Soledad, ossia la prostituzione giovanile, alimentata sull'isola non solo dagli adulti del luogo, ma persino dai turisti occidentali, portatori di un'impeccabile reputazione che invece non è altro che una subdola maschera.

In una continua alternanza di sogno ed incubo, dunque, Angèle Delaunois affronta con delicatezza straordinaria argomenti davvero drammatici, quali il pregiudizio, il miraggio del guadagno facile, l'ineluttabilità di una scelta discutibile, ma che per molti rappresenta l'unica alternativa alla povertà. La sua narrazione è intensa e coinvolgente, ma mai pesante, caratterizzata da svariate sfaccettature, che talvolta la rendono tenera ed ironica, talvolta più seria e riflessiva.

5 E questa pur traumatica esperienza farà sì che Nicolas diventi più forte: se nello scenario lucente dei Carabi ha conosciuto per la prima volta il nero pece del vero squallore, infatti, se in un primo momento ha cercato in tutti i modi di negarlo e di non vederlo, arrivando persino ad insultare sa petite Soledad du soleil, son amour et sa blessure, una volta tornato a casa scoprirà dentro di sé, insieme ad una profonda cicatrice, una consapevolezza nuova ed un forte desiderio di protesta, i quali probabilmente lo aiuteranno ad essere un adulto migliore.

6 Potremmo definirlo un romanzo-denuncia, allora, Soledad du Soleil, un romanzo che è quasi una sfida, caratteristica che del resto è propria di tutti i romanzi editi da Pierre Tisseyre. Perché sono libri che scommettono sulla curiosità, sull'intelligenza e sulla sensibilità dei nostri figli, fratelli e nipoti, perché stimolano la fantasia con immaginari lontani, perché spingono adulti e ragazzi a riscoprire la voglia d'interrogarsi e di mettersi in gioco. Con semplicità, senza nascondere i drammi e i conflitti del mondo in cui viviamo, ma cercando insieme un modo per migliorare ciò che non funziona come dovrebbe.

$7 \quad$ E anche quando le storie si spostano in uno scenario del tutto surreale, gli autori non dimenticano mai d'inserire elementi di attualità e di vita vissuta, facendo una scelta matura ed intelligente. In questo ambito non si può non rimanere colpiti da un romanzo in particolare, Les fées d'Espezel, di Claude D'Astous, dove le fate quebecchesi Georgette e Isabelle, rispettivamente la nonna e la nipote adolescente, raggiungono la Francia in compagnia del dottor Eugène Ophessault, al fine accertare l'esistenza di altre fate nel mondo. La ricerca porterà buoni frutti, poiché in un villaggio sperduto del Languedoc-Roussillon troveranno proprio l'ultimo cerchio di fate ancora viventi sulla terra. E per Isabelle sarà una vera e propria rinascita: rifiutata da entrambi i genitori, infatti, troppo occupati a rifarsi una vita dopo il burrascoso divorzio, pulcino imbronciato in perenne lotta con se stessa e col mondo, Isabelle scoprirà che l'amore, la solidarietà, l'amicizia, esistono davvero. È sufficiente solo crederci. Decide allora di restare ad Espezel, dove si sente per la prima volta amata e serena. Le sue sorelle fate saranno la sua famiglia e qui, in questo luogo incantato, intraprenderà un lungo lavoro 
su di sé, finalmente consapevole che per imparare ad amare gli altri deve imparare prima di tutto ad amare se stessa.

Come si può notare, nonostante il contesto evidentemente fantastico, l'attinenza della storia alle problematiche moderne è davvero sorprendente. E si può dire lo stesso anche dei romanzi fantascientifici che ci regalano Les Éditions Pierre Tisseyre, tra i quali vale la pena di citare Le chien du docteur Chenevert, di Diane Bergeron, Les démons de Rapa Nui, di Gilles Devindilis, e infine la saga in più volumi Storine, Les marécages de l'âme, di Frederik D'Anterny. In questi tre romanzi, infatti, i lettori vengono trascinati nei luoghi più diversi: nello studio di uno scienziato pazzo che svolge esperimenti sugli animali e sugli umani, sulla leggendaria isola di Pasqua attraverso una voragine spaziotemporale e addirittura su altri pianeti popolati di mostri terribili. Ma ovunque siano ambientate le vicende, i personaggi sono sempre paladini del buon senso e della giustizia e si trovano alle prese con tematiche di straordinaria attualità, come l'ecologia e il razzismo, lo sfruttamento dei più deboli e l'etica della ricerca scientifica.

9 Schietti e divertenti, talvolta capricciosi e ostinati, com'è tipico della loro età, ma sempre pronti a battersi per senso di giustizia o per salvare un amico, questi personaggi rappresentano la forza e il cuore di tutte queste opere, anche perché identificarsi in essi, per il lettore, è estremamente facile.

10 E che dire di Villeneuve contre Villeneuve, scritto da Pierre Roy? Questo libretto per i più piccoli riesce magistralmente, in sole sessanta pagine, a distruggere la tradizionale distinzione tra i sessi e tutti quei cliché che vogliono le femmine interessate a bambole e make-up e i maschi a macchine e motori. La piccola Tandra, infatti, è davvero una bambina fuori dal comune e sostenuta dal nonno Roméo, che come lei adora le macchine da corsa, porta avanti un grande sogno: diventare l'unica donna al mondo pilota di formula uno.

11 Un trinomio di fantasia, riflessione e attualità, quindi, ai quali si uniscono una tenera ironia e la dimostrazione che si può essere tutto ciò che si vuole: i romanzi Pierre Tisseyre sono tutto questo. Capaci di affrontare qualunque argomento con la delicatezza e lo humour necessari, essi sono sicuramente un ottimo metodo per stimolare la mente degli adolescenti di oggi e per spingerli, magari insieme ai genitori, ad uscire dal torpore e sviluppare carattere e personalità autonomi. 